This is the author's final, peer-reviewed manuscript as accepted for publication. The publisher-formatted version may be available through the publisher's web site or your institution's library.

\title{
On deconvolution methods
}

Alexander G. Ramm, Anahit Galstian

\section{How to cite this manuscript}

If you make reference to this version of the manuscript, use the following information:

Ramm, A. G., \& Galstian, A. (2003). On deconvolution methods. Retrieved from http://krex.ksu.edu

\section{Published Version Information}

Citation: Ramm, A. G., \& Galstian, A. (2003). On deconvolution methods. International Journal of Engineering Science, 41(1), 31-43.

Copyright: Copyright 2002 Elsevier Science Ltd.

Digital Object Identifier (DOI): doi:10.1016/S0020-7225(02)00145-3

Publisher's Link: http://www.sciencedirect.com/science/article/pii/S0020722502001453 
On deconvolution methods,

Internat. Journ. of Engineering Sci., 41, N1, (2003), 31-43 


\title{
On deconvolution methods
}

\author{
Alexander G. Ramm \\ Anahit Galstian \\ Department of Mathematics,Kansas State Univerisity, \\ Manhattan, KS 66506, USA \\ E-mail: ramm@math.ksu.edu \\ http://www.math.ksu.edu/ ${ }^{\mathrm{ramm}}$
}

Subject Classification 45D05, 45L05, 45P05, 65R20, 65R30

Key words: deconvolution, Volterra equations, ill-posed problems

\begin{abstract}
Several methods for solving efficiently the one-dimensional deconvolution problem are proposed. The problem is to solve the Volterra equation $\mathbf{k} u:=$ $\int_{0}^{t} k(t-s) u(s) d s=g(t), \quad 0 \leq t \leq T$. The data, $g(t)$, are noisy. Of special practical interest is the case when the data are noisy and known at a discrete set of times. A general approach to the deconvolution problem is proposed: represent $\mathbf{k}=A(I+S)$, where a method for a stable inversion of $A$ is known, $S$ is a compact operator, and $I+S$ is injective. This method is illustrated by examples: smooth kernels $k(t)$, and weakly singular kernels, corresponding to Abel-type of integral equations, are considered. A recursive estimation scheme for solving deconvolution problem with noisy discrete data is justified mathematically, its convergence is proved, and error estimates are obtained for the proposed deconvolution method.
\end{abstract}

\section{Introduction}

In many applications one models the relation between input signal $u(t)$ and output signal $g(t)$ by the equation

$$
\mathbf{k} u:=\int_{0}^{t} k(t-s) u(s) d s:=k * u=g(t), \quad 0 \leq t \leq T,
$$


where $k(t)$, given for all $t \geq 0$, characterizes the linear system, $k * u$ is the convolution, $u(t)=k(t)=0$ for $t<0$ and the cases $T<\infty$ and $T=\infty$ are both of interest. In practice $g(t)$ is measured with some error, so $g_{\delta}(t)$ is known, $\left\|g_{\delta}(t)-g(t)\right\| \leq \delta$. The norm we use is $L^{2}\left(0, \infty ; e^{-2 \sigma t}\right)$ norm, or $L^{2}(0, T)$, or $L^{\infty}(0, T)$, and the case $T<\infty$ can be reduced to the case $T=\infty$, as we show below.

If the operator $\mathbf{k}$ in (1.1) is considered as an operator on $L^{\infty}(0, T)$, and $\int_{0}^{T}|k(t)| d t<\infty$, then $\mathbf{k}$ is not boundedly invertible, so problem (1.1) is ill-posed. One can see this from the formula $\int_{0}^{t} k(t-s) e^{i n s} d s \rightarrow 0$ as $n \rightarrow \infty$.

If $T<\infty$, one sets $u(s)=0$ for $t>T$ and defines $g(t)$ for $t>T$ as the lefthand side of equation (1.1). If this is done, then (1.1) can be considered as an equation on $(0, \infty)$ and its solution equals to $u(t)$, the solution of $(1.1)$ on $[0, T]$, when $t \in[0, T]$. We assume that $k(t) \not \equiv 0$ and $\int_{0}^{\infty}|k(t)| d t<\infty$. Then (1.1) has at most one solution in $L^{1}[0, T]$ if $k(t) \neq 0$ almost everywhere in $[0, T]$ ([10], p.327). The assumption $\int_{0}^{\infty}|k(t)| d t<\infty$ can be replaced without loss of generality by a weaker assumption $\int_{0}^{\infty} \exp (-\sigma t)|k(t)| d t<\infty$, with an arbitrary large fixed $\sigma>0$. This weaker assumption can be reduced to the original one by changing variables.

A deconvolution method is a method to construct a stable approximation $u_{\delta}(t)$ of the solution $u(t)$ to equation (1.1), given $g_{\delta}$ :

$$
\left\|u_{\delta}(t)-u(t)\right\|:=\eta(\delta) \rightarrow 0 \quad \text { as } \quad \delta \rightarrow 0 .
$$

An operator $R(\delta)$ which constructs such $u_{\delta}$ from $g_{\delta}, u_{\delta}=R(\delta) g_{\delta}$, is called a regularizer (or a regularizing family, since $\delta \rightarrow 0$ ) if (1.2) holds.

There is a large literature on ill-posed problems. General methods for constructing regularizers have been developed. They include variational regularization, iterative regularization, method of quasisolutions, etc [9]. In Section 2 the specific form of equation (1.1) is used for constructing regularizers for equation (1.1). The emphasis is on the causality property of the regularizer. The idea is similar to the one in [6] and [3]. In Section 3 a simple general method to construct regularizers for equation (1.1) is proposed. This method is practically efficient. It is illustrated by two examples in which the results from [2]-[5], and [7] are used. In Section 4 we investigate a recursive algorithm proposed in [1] for solving equation (1.1) with noisy discrete data. Again, the emphasis is on the causality property of the estimate: we use only the data collected up to the time $t$ in order to estimate the signal $u$ at this time. If one would use a variational regularization for constructing a stable estimation of $u(t)$, one would have to use all the data collected on the full time inteval $[0, T]$, and not only on the "current" time interval $[0, t]$. Our analysis is much shorter than in [1] and yields more detailed results. Moreover, In Section 6 we discuss briefly a generalization of these results to the case of operator-valued kernels, which includes, in particular, matrix-valued kernels, that is, systems of Volterra equations. In Section 5 proofs are given. 


\section{A deconvolution method}

Let $K(\lambda):=\int_{0}^{\infty} e^{-\lambda t} k(t) d t$. By capital letters the Laplace transform is denoted. If $u$ solves $(1.1)$ then $U(\lambda)=K^{-1}(\lambda) G(\lambda)$. One has:

$$
u_{\delta}(t):=\frac{1}{2 \pi i} \int_{\sigma-i \infty}^{\sigma+i \infty} e^{\lambda t} K^{-1}(\lambda) G_{\delta}(\lambda) \frac{1}{\left(\frac{\lambda}{N}+1\right)^{m}} d \lambda, \quad \lambda=\sigma+i \mu \in C_{\sigma}
$$

where $C_{\sigma}$ is the contour $\Re \lambda=\sigma, \lambda=\sigma+i \mu, m>0$ is a sufficiently large positive integer and $N>0$ is a large parameter. We do not show the dependence of $u_{\delta}(t)$ on $m$ and $N$ to simplify the notations. We want to prove that one can choose $N=N(\delta) \rightarrow \infty$ as $\delta \rightarrow 0$, such that (1.2) holds. Note that $G_{\delta}(\lambda)=G(\lambda)+W(\lambda)$, where $|W(\lambda)|=\left|\int_{0}^{\infty} e^{-\lambda t} w(t) d t\right| \leq \frac{\delta}{\sigma}$, the noise $w(t)$ satisfies the inequality $|w(t)| \leq$ $\delta, \sigma=\Re \lambda>0$, and $G(\lambda):=\int_{0}^{\infty} e^{-\lambda t} g(t) d t$. When one uses $L^{\infty}(0, T)$ norm one assumes that

$$
K^{-1}(\lambda) G(\lambda) \in L^{1}\left(C_{\sigma}\right)
$$

This is an a priori assumption on $u(t)$.

We assume throughout this paper that:

$$
|K(\lambda)| \geq c|\lambda|^{-a}, \quad \lambda \in C_{\sigma}, \quad a \in \mathbb{R} .
$$

Here and below $c>0$ denote various constants independent of $\delta$ and $N$. The constant $a$ may be negative, but in many applications $a \geq 0$.

Theorem 2.1 If $m>a+0.5$ and (2.3) holds, then there exists $N(\delta) \rightarrow \infty$ as $\delta \rightarrow 0$, such that $(1.2)$ holds with $L^{2}\left(0, \infty ; e^{-2 \sigma t}\right)$ norm.

If (2.2) and (2.3) hold, and $m>a+1$, then (1.2) holds with $L^{\infty}(0, T)$ norm.

If $m>a+1$, (2.3) holds, and

$$
\left|K^{-1}(\lambda) G(\lambda)\right| \leq \frac{c}{1+|\lambda|^{1+d}}, \quad d=\text { const }>0, \lambda \in C_{\sigma},
$$

then $N(\delta)=O\left(\delta^{-\frac{1}{q}}\right), q=a+d+1, \eta(\delta)=O\left(\delta^{\frac{d}{q}}\right)$ if $0<d<1$, and the norm in (1.2) is $L^{\infty}(0, T)$ norm.

$$
\begin{aligned}
& \text { If } d>1 \text {, then } N(\delta)=O\left(\delta^{-\frac{1}{2+a}}\right) \text { and } \eta(\delta)=O\left(\delta^{\frac{1}{a+2}}\right) . \\
& \text { If } d=1 \text {, then } N(\delta)=O\left(\exp \left[\frac{|\log \delta|}{a+2}(1+o(1)]\right) \text {, and } \eta(\delta)=O\left(\frac{|\log \delta|}{\exp \left[\frac{|\log \delta|}{a+2}(1+o(1)]\right.}\right)\right. \text {. }
\end{aligned}
$$

We sketch proofs in the last Section. 


\section{A general approach to deconvolution}

Suppose the operator $\mathbf{k}$ in (1.1) can be decomposed into a sum $\mathbf{k}:=A+B$, where $A^{-1} B:=S$ is compact in the Banach space $X$, in which $\mathbf{k}$ acts, and $I+S$ is boundedly invertible, or which is the same by the Fredholm alternative, $N(I+S)=\{0\}$, where $N(A)$ is the null space of $A$. In this case $I+S$ is an isomorphism of $X$ onto $X$, $\mathcal{R}(A)=\mathcal{R}(\mathbf{k})$, and

$$
\mathbf{k} u=A(I+S) u=g .
$$

If a regularizer for $A$ is known, then (3.1) can be solved stably by the scheme

$$
u_{\delta}=(I+S)^{-1} R(\delta) g_{\delta},
$$

and (1.2) holds.

Since $I+S$ is an isomorphism, the error $\left\|v-v_{\delta}\right\|$ of the approximation of the stable solution of the equation $A v=g$ by the formula $v_{\delta}=R(\delta) g_{\delta}$ is of the same order as $\left\|u_{\delta}-u\right\|$.

Example 3.1. Let $k(t) \in C^{1}(0, T)$ and assume $k(0) \neq 0$. Then, without loss of generality, one may assume $k(0)=1$. Write (1.1) as

$$
\mathbf{k} u=\int_{0}^{t} u(s) d s+\int_{0}^{t}[k(t-s)-1] u(s) d s:=A u+B u=g .
$$

Here stable inversion of $A$ is equivalent to stable numerical differentiation of noisy data. This problem has been solved in [2] (see also [3] - [5], [7]), and the results of these works yield the following theorem:

Theorem 3.1 Assume that $\|u\|_{W^{2, \infty}} \leq m_{2}<\infty$. Then the operator

$$
R(\delta) g_{\delta}:=\frac{g_{\delta}(t+h(\delta))-g(t)}{h(\delta)}
$$

is a regularizer for the operator $A$ if $h(\delta)=2\left(\frac{\delta}{m_{2}}\right)^{1 / 2}$. One has: $\| R(\delta) g_{\delta}-$ $u \|_{L_{[0, T-h]}^{\infty}} \leq 2 \sqrt{m_{2} \delta}$.

In [5] weaker a priori assumption on $u$ is used: $\|u\|_{a} \leq m_{a}, 0<a \leq 1$, where the Hoelder-space norm is defined as $\|u\|_{a}:=\sup _{x \neq y, x, y \in[0, T]} \frac{|u(t)-u(s)|}{|t-s|^{a}}+\sup _{0 \leq t \leq T}|u(t)|$.

In this example our method yields the equation

$$
(I+S) u_{\delta}=R(\delta) g_{\delta}
$$

where $S$ is a Volterra operator: $S u_{\delta}:=\int_{0}^{t} k^{\prime}(t-s) u_{\delta}(s) d s$. Therefore $u_{\delta}$ can be easily found by iterations. 
Example 3.2. Let $k(t)=\frac{t^{-\gamma}}{\Gamma(1-\gamma)}+m(t), 0<\gamma<1, m(t) \in C^{1}$,

$$
A u=\frac{t^{-\gamma}}{\Gamma(1-\gamma)} * u, \quad B u=m * u
$$

One has $A^{-1} g=\frac{1}{\Gamma(\gamma)} \int_{0}^{t} \frac{g^{\prime}(s) d s}{(t-s)^{1-\gamma}}$. Define

$$
R_{1}(\delta) g_{\delta}:=\frac{1}{\Gamma(\gamma)} \int_{0}^{t} \frac{\left(R(\delta) g_{\delta}\right)(s)}{(t-s)^{1-\gamma}} d s
$$

where $R(\delta)$ is defined in Theorem 3.1.

Theorem 3.2 The operator-function $R_{1}(\delta)$ is a regularizer for equation $A u=g$, the operator $S:=A^{-1} B$ is compact in $L^{2}(0, T)$ and $N(I+S)=\{0\}$.

Compactness of $S$ is clear from its definition. The operator $R_{1}(\delta)$ is a regularizer for $A$ because $R(\delta)$ is a regularizer for the operator of differentiation. Finally $N(I+S)=$ $\{0\}$ because $A^{-1} B$ is a Volterra operator. Therefore $u_{\delta}$ can be easily computed by our general method and (1.2) holds.

\section{Recursive estimation given discrete noisy data}

Assume that

$$
g_{\delta}(n h):=\xi_{n}=g(n h)+w_{n}
$$

are noisy measurements of the data $g(t)$ at the time moments $n h, h>0$ is small number, $\left|w_{n}\right| \leq \delta$ is noise. One wishes to estimate stably $u(t)$, the solution to (1.1), given the data $\xi_{1}, \ldots \xi_{n}$. The following estimation method was essentially proposed in [1]. Set $v_{0}=\frac{\xi_{1}-\xi_{0}}{h}$. Define $v_{j}$ by recursive formulas:

$$
\alpha v_{n}+\sum_{j=0}^{n-1} \int_{j h}^{(j+1) h} k(n h-s) d s v_{j}=\xi_{n}, \quad v_{0}=\frac{\xi_{1}-\xi_{0}}{h} .
$$

In this Section we assume that $k(t) \in L^{1}\left(\mathbb{R}_{+}\right) \cap L_{\text {loc }}^{2}\left(\mathbb{R}_{+}\right)$and $u(t) \in L_{\text {loc }}^{2}\left(\mathbb{R}_{+}\right)$. Then $g(t) \in C_{l o c}\left(\mathbb{R}_{+}\right)$, as the following lemma claims.

Lemma 4.1 If $u, k \in L_{\text {loc }}^{2}\left(\mathbb{R}_{+}\right)$then $k * u \in C_{\text {loc }}\left(\mathbb{R}_{+}\right)$.

Proof. One has

$$
\begin{aligned}
|(k * u)(t+h)-(k * u)(t)| & \leq \int_{0}^{t+h}|k(t+h-s)-k(t-s)||u| d s \\
& +\int_{t}^{t+h}|k(t-s)||u| d s:=I_{1}+I_{2} .
\end{aligned}
$$


Now,

$$
I_{1}^{2} \leq \int_{0}^{t+h}|k(t+h-s)-k(t-s)|^{2} d s \int_{0}^{t+h}|u|^{2} d s \rightarrow 0 \text { as } h \rightarrow 0
$$

and

$$
I_{2}^{2} \leq \int_{t}^{t+h}|k(t-s)|^{2} d s \int_{t}^{t+h}|u(s)|^{2} d s \rightarrow 0 \text { as } h \rightarrow 0 .
$$

Lemma 4.1 is proved.

This lemma makes it reasonable to assume that $g(t) \in C_{l o c}\left(\mathbb{R}_{+}\right)$. In [1] the case $g \in L_{l o c}^{2}\left(\mathbb{R}_{+}\right)$is discussed, when $g$ is not defined pointwise. It is proposed in [1] to use a mollification of $g$ around the points $n h$ instead of using $g(n h)$. However, this mollification requires a knowledge of $g$ in a neighborhoods of all points $n h$, and this is an information different from the one assumed at the beginning, namely $\xi_{1}, \ldots \xi_{n}$. By this reason and because of Lemma 4.1, we assume that $g(t) \in C_{\text {loc }}\left(\mathbb{R}_{+}\right)$, the space of functions continuous on any compact subinterval of $\mathbb{R}_{+}$.

Our assumptions in this section are:

A) $k(t) \in L^{1}\left(\mathbb{R}_{+}\right) \cap L_{\text {loc }}^{2}\left(\mathbb{R}_{+}\right)$and $u(t) \in L_{\text {loc }}^{2}\left(\mathbb{R}_{+}\right)$,

B) (2.3) holds,

C) the union of the spectra of $\{K(\lambda)\}_{\forall \lambda \in C_{\sigma}}$ does not contain the set $\{z: z \in$ $\mathbb{C}, \pi-\varphi<\arg z<\pi+\varphi,|z|<r\}$, where $\varphi>0$ and $r>0$ are arbitrary small fixed numbers.

If the assumptions A), B), and C) hold, then our result is Theorem 4.1 below. If, in addition, $k$ and $g$ are Hoelder-continuous, then our result is Theorem 4.2 below, which gives the rate of convergence in (1.2).

We prove that if $\alpha=\alpha(\delta)$ and $h=h(\delta)$ are chosen suitably, then the function $v_{\delta}(t)$, defined by the formula:

$$
v_{\delta}(t)=v_{j} \quad \text { for } \quad j h \leq t \leq(j+1) h,
$$

approximates stably $u(t)$, so that $\left\|v_{\delta}(t)-u(t)\right\| \rightarrow 0$ as $\delta \rightarrow 0$, where $\|\cdot\|$ is $L^{\infty}(0, T)$ norm. The rate of convergence is estimated in Theorem 4.2 under additional a priori assumptions.

Let

$$
\alpha u_{\alpha}(t)+\int_{0}^{t} k(t-s) u_{\alpha}(s) d s=g(t), \quad 0 \leq t \leq T .
$$

The function $v_{\delta}(t)$, defined above, solves the equation

$$
\alpha v_{\delta}(t)+\int_{0}^{t} k(t-s) v_{\delta}(s) d s=f_{\delta}(t)
$$


where $f_{\delta}(j h)=\xi_{j}$ for $t=j h$, and for other values of $t$ the function $f_{\delta}(t)$ is defined as the left-hand side of (4.3):

$$
f_{\delta}(t):=g_{\delta}(n h)-g(t)+\int_{0}^{t}[k(t-s)-k(n h-s)] v_{\delta}(s) d s+g(t):=\varphi_{\delta}(t)+g(t),
$$

where $(n-1) h \leq t \leq n h, \quad n=1,2,3, \ldots \ldots$

One has

$$
\left|\varphi_{\delta}(t)\right| \leq \delta+\gamma_{g}(h)+c(T)\left\|v_{\delta}\right\| \gamma_{k}(h)
$$

where

$\gamma_{g}(h):=|g(n h)-g(t)| \rightarrow 0, \quad \gamma_{k}(h):=\int_{0}^{t}|k(t-s)-k(n h-s)| d s \rightarrow 0 \quad$ as $h \rightarrow 0$, and $(n-1) h \leq t \leq n h, n=1,2,3 \ldots \ldots$

Denote $v_{\delta}-u_{\alpha}:=w$. From (4.2) and (4.3) one gets: $w=(\alpha+\mathbf{k})^{-1} \varphi_{\delta}$, where $\alpha+\mathbf{k}:=\alpha I+\mathbf{k}$, and $I$ is the identity operator. One has $\left\|(\alpha+\mathbf{k})^{-1}\right\| \leq c \alpha^{-1}$, and $\left\|\varphi_{\delta}\right\| \leq \delta+\gamma_{g}(h)+c(T) \gamma_{k}(h)\left\|v_{\delta}\right\|$. Therefore:

$$
\left\|v_{\delta}-u_{\alpha}\right\| \leq c(T) \frac{\delta+\gamma_{g}(h)+c(T) \gamma_{k}(h)\left\|v_{\delta}\right\|}{\alpha} .
$$

Let us choose $h=h(\delta)$ such that $\frac{\gamma_{k}(h)+\gamma_{g}(h)}{\alpha} \rightarrow 0$ as $\alpha \rightarrow 0$, and $\alpha=\alpha(\delta)$ so that $\frac{\delta}{\alpha(\delta)} \rightarrow 0$ as $\delta \rightarrow 0$

Let $u_{\delta}:=u_{\alpha(\delta)}$. Then (1.2) holds by Lemma 5.1 below:

$\left\|u_{\delta}-u\right\|=\left\|u_{\alpha(\delta)}-u\right\|=\left\|(\alpha+\mathbf{k})^{-1} \mathbf{k} u-u\right\|:=\varepsilon(\alpha) \rightarrow 0$ as $\alpha:=\alpha(\delta) \rightarrow 0$.

From (4.3) one gets

$$
v_{\delta}=(\alpha+\mathbf{k})^{-1} g+(\alpha+\mathbf{k})^{-1}\left[g_{\delta}(n h)-g(t)\right]+(\alpha+\mathbf{k})^{-1} \mathbf{Q} v_{\delta},
$$

where

$$
\mathbf{Q} v_{\delta}:=\int_{0}^{t}[k(t-s)-k(n h-s)] v_{\delta} d s .
$$

Since $\left\|(\alpha+\mathbf{k})^{-1}\right\| \leq \frac{c}{\alpha}$ and $\left\|g_{\delta}(n h)-g(t)\right\| \leq c(T)\left(\delta+\gamma_{g}(h)\right)$, one has:

$$
\left\|(\alpha+\mathbf{k})^{-1}\left[g_{\delta}(n h)-g(t)\right]\right\| \leq c(T) \frac{\delta+\gamma_{g}(h)}{\alpha} \rightarrow 0 \quad \delta \rightarrow 0,
$$

provided that $\alpha=\alpha(\delta)$ and $h=h(\delta)$ are chosen so that $\frac{\delta}{\alpha(\delta)} \rightarrow 0$ and $\frac{\gamma_{g}(h(\delta))}{\alpha(\delta)} \rightarrow 0$ as $\delta \rightarrow 0$. Let $\alpha=\alpha(\delta)$. Then $\left\|(\alpha+\mathbf{k})^{-1} \mathbf{Q} v_{\delta}\right\| \leq \frac{c}{\alpha} \gamma_{k}(h)\left\|v_{\delta}\right\|$, and if $\frac{\gamma_{k}(h(\delta))}{\alpha(\delta)} \rightarrow 0$ as $\delta \rightarrow 0$, then $\left\|(\alpha+\mathbf{k})^{-1} \mathbf{Q}\right\| \leq \frac{c \gamma_{k}(h)}{\alpha} \rightarrow 0$ as $\delta \rightarrow 0$. Thus

$$
v_{\delta}=\left[I-(\alpha+\mathbf{k})^{-1} \mathbf{Q}\right]^{-1}\left[(\alpha+\mathbf{k})^{-1} g+O\left(\frac{\delta+\gamma_{g}(h)}{\alpha}\right)\right],
$$


where $O\left(\frac{\delta+\gamma_{g}(h)}{\alpha}\right)$ denotes an element whose norm is $O\left(\frac{\delta+\gamma_{g}(h)}{\alpha}\right)$. Therefore, with $u_{\delta}:=(\alpha(\delta)+\mathbf{k})^{-1} g$, one gets:

$$
\left\|v_{\delta}-u_{\delta}\right\| \leq c(T) \frac{\delta+\gamma_{g}(h)+\gamma_{k}(h)}{\alpha} .
$$

Consequently:

$\left\|v_{\delta}-u\right\| \leq\left\|v_{\delta}-u_{\delta}\right\|+\left\|u_{\delta}-u\right\| \leq c(T) \frac{\delta+\gamma_{g}(h)+\gamma_{k}(h)}{\alpha}+\varepsilon(\alpha) \rightarrow 0 \quad$ as $\quad \delta \rightarrow 0$.

We have proved:

Theorem 4.1 Assume A), B), and $\mathbf{C})$. Then there exist $h=h(\delta) \rightarrow 0$ and $\alpha=$ $\alpha(\delta) \rightarrow 0$ such that (4.5) holds.

If $g(t)$ and $k(t)$ are Hoelder-continuous, then $\gamma_{g}(h)+\gamma_{k}(h)=O\left(h^{b}\right), 0<b \leq 1$. Put $\delta=h^{b}$. Then (4.5) can be written as:

$$
\left\|v_{\delta}-u\right\| \leq c\left[\frac{\delta}{\alpha}+\varepsilon(\alpha)\right]
$$

Let us estimate $\varepsilon(\alpha)=\left\|u_{\delta}-u\right\|$. One has:

$$
\begin{aligned}
\varepsilon(\alpha) & =\int_{-\infty}^{\infty}\left|\alpha(\alpha+K(\lambda))^{-1}\right|\left|K^{-1}(\lambda) G(\lambda)\right| d \mu \\
& \leq \int_{-\infty}^{\infty} \alpha\left|(\alpha+K(\lambda))^{-1}\right| \frac{d \mu}{\left[1+\left(\sigma^{2}+\mu^{2}\right)^{1 / 2}\right]^{1+d}} \\
& =\int_{-M}^{M}+\int_{-\infty}^{M}+\int_{M}^{\infty}:=I_{1}+I_{2}+I_{3} .
\end{aligned}
$$

Using (2.3), one gets:

$$
I_{1} \leq c \alpha \int_{-M}^{M} \frac{\mu^{a} d \mu}{(1+|\mu|)^{d+1}} \leq c \alpha M^{a-d}
$$

The estimates of $I_{2}$ and $I_{3}$ are similar. Let us estimate, for example, $I_{3}$ :

$$
I_{3} \leq c \int_{M}^{\infty} \frac{d \mu}{(1+\mu)^{d+1}} \leq c M^{-d}, \quad d>0 .
$$

Thus

$$
\varepsilon(\alpha) \leq c\left(\alpha M^{a-d}+M^{-d}\right) .
$$

If $d \geq a$, then $\varepsilon(\alpha) \leq c \alpha,\left\|v_{\delta}-u\right\| \leq c\left[\frac{\delta}{\alpha}+\varepsilon(\alpha)\right]$. Minimizing with respect to $\alpha$, one gets $\left\|v_{\delta}-u\right\| \leq c \delta^{1 / 2}$ if $\alpha=\delta^{1 / 2}$. If $d<a$, then $\varepsilon(\alpha) \leq c\left(\alpha M^{a-d}+M^{-d}\right)$. Minimizing with respect to $M$, one gets $\varepsilon(\alpha) \leq c \alpha^{d / a},\left\|v_{\delta}-u\right\| \leq c\left(\delta \alpha^{-1}+\alpha^{d / a}\right)$. Minimizing with respect to $\alpha$, one gets $\left\|v_{\delta}-u\right\| \leq c \delta^{\frac{d}{d+a}}$.

Let us summarize the result: 
Theorem 4.2 Assume A), B), and C). If $g$ and $k$ are Hoelder-continuous, so that $\gamma_{g}(h)+\gamma_{k}(h)=O\left(h^{b}\right), 0<b \leq 1$, then $\left\|v_{\delta}-u\right\| \leq c \delta^{\frac{d}{d+a}}$, provided that $h=\delta^{\frac{1}{b}}$, $\alpha=O\left(\delta^{\frac{a}{d+a}}\right)$, and $d<a$. If $d \geq a$, then $\left\|v_{\delta}-u\right\| \leq c \delta^{\frac{1}{2}}$.

In [8] a singular perturbation problem was solved for a class of one- and multidimensional integral equations. The problem we study in Sec. 4 contains a singular perturbation problem as a basic component: we are interested in the behavior of the operator $(\alpha+\mathbf{k})^{-1}$ as $\alpha \rightarrow+0$.

\section{Proofs.}

The norm below is $L^{2}\left(0, \infty ; e^{-2 \sigma t}\right)$ norm, it is equivalent to $L^{2}(0, T)$ norm on $(0, T)$. By the spectrum of a scalar function $K(\lambda)$ we mean the set of its values, and if $K(\lambda)$ is an operator-valued function, then its spectrum is defined as usual.

Lemma 5.1 Let (2.3) hold and assume that the union of the spectra of $\{K(\lambda)\}_{\forall \lambda \in C_{\sigma}}$ does not contain the set $\{z: z \in \mathbb{C}, \pi-\varphi<\arg z<\pi+\varphi,|z|<r\}$, where $\varphi>0$ and $r>0$ are arbitrary small fixed numbers. Then

$$
\varepsilon(\alpha):=\left\|(\alpha+\mathbf{k})^{-1} \mathbf{k} u-u\right\| \rightarrow 0 \quad \text { as } \quad \alpha \rightarrow+0 \quad \text { for all } \quad u \in L^{2}\left(0, \infty ; e^{-2 \sigma t}\right) .
$$

Proof. One has

$$
\begin{aligned}
& \left\|(\alpha+\mathbf{k})^{-1} \mathbf{k} u-\mathbf{k}^{-1} \mathbf{k} u\right\|^{2}=\left\|(\alpha+\mathbf{k})^{-1} \alpha \mathbf{k}^{-1} \mathbf{k} u\right\|^{2} \\
& =\left\|\alpha(\alpha+\mathbf{k})^{-1} u\right\|^{2}=\left\|\frac{e^{\sigma t}}{2 \pi} \int_{-\infty}^{\infty} e^{i \mu t} \alpha(\alpha+K(\lambda))^{-1} K^{-1}(\lambda) G(\lambda) d \mu\right\|^{2} \\
& =\frac{1}{2 \pi} \int_{-\infty}^{\infty} \alpha^{2}\left|(\alpha+K(\lambda))^{-1}\right|^{2}\left|K^{-1}(\lambda) G(\lambda)\right|^{2} d \mu:=\varepsilon(\alpha) \rightarrow 0 \quad \text { as } \quad \alpha \rightarrow 0, \\
& \lambda=\sigma+i \mu \in C_{\sigma} .
\end{aligned}
$$

Here we have used: 1) Parseval's equality; 2) the asumption $u \in L^{2}\left(0, \infty ; e^{-2 \sigma t}\right)$ which is equivalent to $\left.K^{-1}(\lambda) G(\lambda) \in L^{2}\left(C_{\sigma}\right) ; 3\right)$ the estimate $\sup _{\lambda \in C_{\sigma}}\left|\alpha(\alpha+K(\lambda))^{-1}\right|$ $\leq c$ which follows from the assumption about the range of $K(\lambda)$ on $\left.C_{\sigma} ; 4\right)$ estimate $(2.3)$; and 5$)$ the dominated convergence theorem. Lemma 5.1 is proved

Lemma 5.2 Under the assumptions of Lemma 5.1 one has:

$$
\left\|(\alpha+\mathbf{k})^{-1} g_{\delta}-u\right\| \leq \frac{c \delta}{\alpha}+\varepsilon(\alpha), \quad \varepsilon(\alpha) \rightarrow 0 \quad \text { as } \quad \alpha \rightarrow 0, \quad c=\text { const }>0 .
$$


Proof. One has

$\left.\left\|(\alpha+\mathbf{k})^{-1} g_{\delta}-u\right\| \leq\left\|(\alpha+\mathbf{k})^{-1}\left(g_{\delta}-g\right)\right\|+\left\|(\alpha+\mathbf{k})^{-1} g-u\right\| \leq \|(\alpha+\mathbf{k})^{-1}\right) \| \delta+\varepsilon(\alpha)$.

By Lemma 5.1, $\varepsilon(\alpha) \rightarrow 0$ as $\alpha \rightarrow 0$, and $\left\|(\alpha+\mathbf{k})^{-1}\right\| \leq c \alpha^{-1}$, where $c=$ const $>0$ depends on $\varphi$, as follows from the proof of Lemma 5.1 and from the estimate

$$
\sup _{\lambda \in C_{\sigma}}\left|(\alpha+K(\lambda))^{-1}\right| \leq \frac{c}{\alpha} .
$$

Lemma 5.2 is proved.

Proof of Theorem 2.1. If $u_{\delta}$ is defined in $(2.1)$ and $\|\cdot\|$ is $L^{2}\left(0, \infty ; e^{-2 \sigma t}\right)$ norm, then Parseval's equality yields

$$
\begin{aligned}
& \left\|u_{\delta}-u\right\|^{2}=\frac{1}{2 \pi} \int_{-\infty}^{\infty}\left|K^{-1}(\lambda) G_{\delta}(\lambda) \frac{1}{\left(\frac{\lambda}{N}+1\right)^{m}}-K^{-1}(\lambda) G(\lambda)\right|^{2} d \mu \\
& \leq \frac{1}{\pi} \int_{-\infty}^{\infty}\left|K^{-1}(\lambda) G(\lambda)\right|^{2}\left|\frac{1}{\left(\frac{\lambda}{N}+1\right)^{m}}-1\right|^{2} d \mu+\frac{\delta^{2}}{\pi \sigma^{2}} \int_{-\infty}^{\infty} \frac{\left|K^{-1}(\lambda)\right|^{2}}{\left|\frac{\lambda}{N}+1\right|^{2 m}} d \mu \\
& :=I_{1}+\delta^{2} I_{2},
\end{aligned}
$$

where we have used the formulas $G_{\delta}=G+W,|W| \leq \frac{\delta}{\sigma}$.

If $N \rightarrow \infty$ then $I_{1}=I_{1}(N) \rightarrow 0$ by the dominated convergence theorem. Let us estimate $I_{2}$ assuming (2.3) and taking $m-a>0.5$ :

$$
\begin{aligned}
I_{2} & \leq c N^{2 m} \int_{-\infty}^{\infty} \frac{\left(\sigma^{2}+\mu^{2}\right)^{a} d \mu}{\left[(\sigma+N)^{2}+\mu^{2}\right]^{m}} \leq c N^{2 m} \int_{-\infty}^{\infty} \frac{d \mu}{\left[(\sigma+N)^{2}+\mu^{2}\right]^{m-a}} \\
& \leq c \frac{N^{2 m}}{(N+\sigma)^{2 m-2 a-1}} \int_{-\infty}^{\infty} \frac{d \nu}{\left(1+\nu^{2}\right)^{m-a}} \leq c N^{2 a+1} .
\end{aligned}
$$

Thus, if $2 a+1>0$, then, using the estimate $(x+y)^{1 / 2} \leq x^{1 / 2}+y^{1 / 2}, x, y \geq 0$, one gets:

$$
\left\|u_{\delta}-u\right\| \leq \delta c^{1 / 2} N^{a+\frac{1}{2}}+I_{1}^{\frac{1}{2}}(N):=\eta(\delta, N) .
$$

Minimizing $\eta(\delta, N)$ with respect to $N$ for a fixed $\delta$, denoting the minimizer by $N(\delta)$, $N(\delta) \rightarrow \infty$, as $\delta \rightarrow 0$, and the minimum by $\eta(\delta):=\eta(\delta, N(\delta))$, one gets $\eta(\delta) \rightarrow 0$ as $\delta \rightarrow 0$. Thus, (1.2) is proved with $L^{2}\left(0, \infty ; e^{-2 \sigma t}\right)$ norm.

If $L^{\infty}(0, T)$ norm is used for $\left\|u(t)-u_{\delta}(t)\right\|$, then one gets (1.2) if assumptions (2.2) and (2.3) are used. Namely,

$$
\begin{aligned}
\sup _{0 \leq t \leq T}\left|u_{\delta}(t)-u(t)\right| & \leq \frac{\exp (\sigma T)}{2 \pi} \int_{-\infty}^{\infty}\left|K^{-1} G_{\delta}\left(1+\frac{\lambda}{N}\right)^{-m}-K^{-1} G\right| d \mu \\
& \leq c(T)\left(J_{1}+J_{2}\right):=\eta
\end{aligned}
$$


where

$$
J_{1}:=J_{1}(N)=\int_{-\infty}^{\infty}\left|K^{-1} G\right|\left|\left(1+\frac{\lambda}{N}\right)^{-m}-1\right| d \mu
$$

and

$$
J_{2}:=J_{2}(N)=\frac{\delta}{\sigma} \int_{-\infty}^{\infty}\left|K^{-1}\right|\left|1+\frac{\lambda}{N}\right|^{-m} d \mu .
$$

If $K^{-1} G \in L^{1}\left(C_{\sigma}\right)$, then $\lim _{N \rightarrow \infty} J_{1}=0$ by the dominated convergence theorem. If $m>a+1$ and (2.3) holds, then

$$
J_{2} \leq c \delta N^{a+1}
$$

Thus (1.2) holds with $L^{\infty}(0, T)$-norm provided that $K^{-1} G \in L^{1}\left(C_{\sigma}\right), m>a+1$ and (2.3) holds.

If (2.4) holds, one can get a rate of decay. Namely

$$
J_{1}=\int_{-M}^{M}+\int_{|\mu|>M}=j_{1}+j_{2},
$$

where

$$
\begin{gathered}
j_{1} \leq \int_{-M}^{M}\left(1+|\lambda|^{d+1}\right)^{-1}\left|\left(1+\frac{\lambda}{N}\right)^{-m}-1\right| d \mu \\
\leq c \int_{0}^{M}\left(1+|\lambda|^{d+1}\right)^{-1} \frac{|\lambda|}{N} d \mu \leq c M^{1-d} N^{-1}, \text { if } 0<d<1 \\
j_{1} \leq c N^{-1} \text { if } d>1, \\
j_{1} \leq c \frac{\log M}{N} \text { if } d=1,
\end{gathered}
$$

and

$$
j_{2} \leq c \int_{M}^{\infty}\left(1+|\lambda|^{d+1}\right)^{-1}\left|\left(1+\frac{\lambda}{N}\right)^{-m}-1\right| d \mu \leq c \int_{M}^{\infty}\left(1+|\lambda|^{d+1}\right)^{-1} \leq c M^{-d},
$$

$c>0$ stands for various constants, and $\lambda=\sigma+i \mu$. Thus

$$
\begin{gathered}
J_{1} \leq c\left(M^{1-d} N^{-1}+M^{-d}\right) \text { if } 0<d<1, \\
J_{1} \leq c\left(N^{-1}+M^{-d}\right) \text { if } d>1, \\
J_{1} \leq c\left(\frac{\log M}{N}+M^{-d}\right) \text { if } d=1 .
\end{gathered}
$$

If $0<d<1$ then choose $M=N$ and get $J_{1} \leq c N^{-d}$. Therefore, if $m>a+1$ and (2.3) and (2.4) hold, then

$$
J_{1}+J_{2} \leq c\left(\delta N^{a+1}+N^{-d}\right) \text { if } 0<d<1 .
$$


Minimizing with respect to $\mathrm{N}$ for a fixed $\delta>0$, one gets the minimizer $N=N(\delta)=$ $O\left(\delta^{-\frac{1}{1+a+d}}\right)$ and the estimate $\eta \leq O\left(\delta^{\frac{d}{1+a+d}}\right)$ if $0<d<1$. If $d>1$ then choose $M^{d}=N$ and get $J_{1} \leq c N^{-1}, J_{1}+J_{2} \leq c\left(\delta N^{a+1}+N^{-1}\right), N(\delta)=O\left(\delta^{-\frac{1}{2+a}}\right)$, and the estimate $\eta \leq O\left(\delta^{\frac{1}{2+a}}\right)$. If $d=1$ then choose $M=N$ and get $J_{1} \leq c \frac{\log N}{N}$, $J_{1}+J_{2} \leq c\left(\delta N^{a+1}+\frac{\log N}{N}\right), N(\delta)=O\left(\exp \left[\frac{|\log \delta|}{a+2}(1+o(1)]\right)\right.$, and the estimate $\eta \leq$ $O\left(\frac{|\log \delta|}{\exp \left[\frac{\log \delta \mid}{2+a}(1+o(1))\right]}\right)$. Theorem 2.1 is proved.

Remark 5.3. If $k(t)>0$ and $k(t) \rightarrow 0$ monotonically, then $\Re K(\lambda) \geq 0$ for $\Re \lambda>0$. If $\Re K(\lambda) \geq 0$ then $\left|\alpha(\alpha+K(\lambda))^{-1}\right| \leq 1$. Condition $\Re K(\lambda) \geq 0$ implies that the assumption of Lemma 5.1 holds with $\varphi=\frac{\pi}{2}$ and $r=\infty$.

\section{Generalizations.}

Most of our results and proofs remain valid for operator-valued functions $k(t)$, in particular for matrix-valued kernels, that is, for systems of Volterra equations. Let $k(t)$ be an operator in a Banach space, and $K(\lambda)$ be its Laplace transform. If one replaces the absolute values by the norms in (2.3), (2.4) and elsewhere in the proofs, then one gets Theorems 2.1, 3.1, 3.2, and 4.1 and lemmas 5.1 and 5.2 with operator-valued $k(t)$.

Acknowledgement. AGR thanks Prof. L. Pandolfi and Dr. F. Fagnani for discussions. Their paper [1] was useful for the results in Sec. 4 of this paper.

\section{References}

[1] Fagnani, F., Pandolfi, L., A recursive algorithm for the approximate solutions of Volterra integral Equations of first kind, Preprint, Dipartimento di Matematica, Politecnico Torino, (2002).

[2] Ramm, A.G., On numerical differentiation, Mathem., Izvestija vuzov, 11, (1968), 131-135.

[3] Ramm, A.G., Stable solutions of some ill-posed problems, Math. Meth. in the appl. Sci., 3, (1981), 336-363.

[4] Ramm, A.G., Random fields estimation theory, Longman Scientific and Wiley, New York, 1990.

[5] Ramm, A.G., Inequalities for the derivatives, Math. Ineq. and Appl., 3, N1, (2000), 129-132. 
[6] Ramm, A.G., Optimal harmonic synthesis of generalized Fourier series and integrals with randomly perturbed coefficients, Radiotechnika, 28, (1973), 4449.

[7] Ramm, A.G., Smirnova, A., On stable numerical differentiation, Mathem. of Computation, 70, (2001), 1131-1153.

[8] Ramm, A.G., Shifrin, E., Asymptotics of the solutions to singularly perturbed integral equations II., J. Math. Anal. Appl., 178, N2, (1993), 322-343; Asymptotics of the solutions to singularly perturbed multidimensional integral equations, ibid., 190, N3, (1995), 667-677.

[9] Tikhonov, A. N., Leonov, A. S., Yagola, A. G., Nonlinear ill-posed problems, Vol. 1, 2, Chapman\&Hall, London, 1998.

[10] Titchmarsh E., Introduction to the theory of Fourier Integrals, Clarendon Press, Oxford, 1937. 